\title{
Avaliação dos efeitos miorelaxante, antiespasmódico e antinociceptivo do extrato aquoso da Phoradendron piperoides (Kunt.) Trel. (Viscaceae)
}

\author{
Kellyane S. Dias, Dayse S. Almeida, Aline B. L. Silva, Maxsuel S. Marques, Igor A. \\ C. Menezes, Thiago C. Santos, Iderjane C. M. Mello, Ana C. S. Carvalho, Ângelo R. \\ Antoniolli, Rosilene M. Marçal*
}

\begin{abstract}
Departamento de Fisiologia, Universidade Federal de Sergipe, Av. Marechal Rondon, s/n, Rosa Elze,
\end{abstract} 49100-000, São Cristóvão, SE, Brasil

\begin{abstract}
RESUMO: Foram investigados os efeitos miorelaxante, antiespasmódico e antinociceptivo do extrato aquoso liofilizado das folhas da Phoradendron piperoides. A toxicidade aguda também foi avaliada. No íleo isolado de cobaio, o extrato aquoso da P. piperoides $(0,05-2,0 \mathrm{mg} / \mathrm{mL})$ produziu relaxamento de forma concentração-dependente $\left(\mathrm{IC}_{50}=0,114 \mathrm{mg} / \mathrm{mL}\right)$ e, na concentração de 1,5 $\mathrm{mg} / \mathrm{mL}$, reduziu a amplitude das contrações induzidas por carbacol $(2 \mu \mathrm{M})$, histamina $(2 \mu \mathrm{M}) \mathrm{e}$ $\mathrm{BaCl}_{2}(0,03 \mathrm{M})$ em 46,6; 38,6 e 55,3\% ( $\left.\mathrm{p}<0,001\right)$, respectivamente. Em camundongos, o extrato aquoso liofilizado (100-400 mg/kg) não reduziu de forma significativa as contorções abdominais induzidas por ácido acético, não modificou o tempo de reação dos animais no teste da formalina e não aumentou o tempo de latência ao calor no teste da placa quente. No ensaio de toxicidade aguda utilizado, não foi detectada a morte de nenhum animal após tratamento com doses de até $5 \mathrm{~g} / \mathrm{kg}$ (p.o.) do extrato. Em conclusão, os resultados obtidos indicam que o extrato aquoso da $P$. piperoides apresenta efeito antiespasmódico e baixa toxicidade aguda. O extrato, no entanto, não possui efeito antinociceptivo.
\end{abstract}

Unitermos: Phoradendron piperoides, Viscaceae, antinociceptivo, antiespasmódico, miorelaxante, toxicidade.

\begin{abstract}
Investigation on miorelaxant, antispasmodic and analgesic effects of the aqueous extract of Phoradendron piperoides". The present work evaluated the antinociceptive, miorelaxant and antispasmodic effects as well as the acute toxicity of the aqueous extract from leaves of Phoradendron piperoides. In guinea pig ileum, the plant extract $(0.05-2.0 \mathrm{mg} / \mathrm{kg})$ decreased the preparations basal tone in a dose-dependent manner $\left(\mathrm{IC}_{50}=0.114 \mathrm{mg} / \mathrm{mL}\right)$ and it $(1.5 \mathrm{mg} / \mathrm{mL})$ reduced $(\mathrm{p}<0.001)$ the contractions induced by carbachol $(2 \mu \mathrm{M})$, histamine $(2$ $\mu \mathrm{M})$ and $\mathrm{BaCl}_{2}(0.03 \mathrm{M})$. The extract, at oral doses of 100,200 , and $400 \mathrm{mg} / \mathrm{kg}$, did not manifest a significant antinociceptive effect in the writhing, formalin and hot-plate tests. Moreover, no animal deaths were observed in doses up to $5 \mathrm{~g} / \mathrm{kg}$. In conclusion, the aqueous extract of Phoradendron piperoides showed no antinociceptive effect and no acute toxicity in mice. Indeed, it revealed miorelaxant and antispasmodic activities that are probably miogenic and not specific for neurotransmitters.
\end{abstract}

Keywords: Phoradendron piperoides, Viscaceae, miorelaxant, antispasmodic, antinociceptive, acute toxicity.

\section{INTRODUÇÃO}

Phoradendron sp apresentam indicação popular no tratamento de dor, distúrbios gastrointestinais e "doenças femininas" dentre outras (Timothy, 1999; Agra et al., 2007). Diversas plantas deste gênero têm sido estudadas tanto na fitoquímica quanto na farmacologia. A análise fitoquímica da Phoradendron liga mostrou que esta espécie produz Cglicosilflavonas e 3-desoxiproantocianidina (Varela et al., 2004). Phoradendron classifolium exerce atividade citoproterora comparável à atropina e $P$. latifolium causa contração no ducto deferente de cobaios (Queiroz-Neto; Melito, 1990; Gonzales et al., 2000). Atividade antitumoral foi detectada para $P$. liga e P. tomentosum e anti-HIV para P. juniperinum (Kashiwada et al., 1998; Li et al., 2002; Johansson et al., 2003).

Phoradendron piperoides (Kunt.) Trel. (Viscaceae), uma planta hemiparasita, é popularmente conhecida como "Enxerto-de-passarinho" no estado de 
Sergipe, sendo a infusão de suas folhas utilizada como antibiótico, abortivo e no combate à dor. Apesar do uso popular no combate à dor, não foram encontrados na literatura trabalhos que investigassem este efeito. Considerando estes fatos, o objetivo inicial deste trabalho foi investigar o efeito analgésico sugerido pelo uso popular. A toxicidade aguda e a resposta do extrato em íleo de cobaio foram também avaliadas.

\section{MATERIAL E MÉTODO}

\section{Material botânico}

Foram utilizadas folhas frescas de Phoradendron piperoides coletadas na cidade de São Cristóvão (10 $56^{\prime} \mathrm{S}$; $\left.37^{\circ} 05^{\prime} \mathrm{W}\right)$, Sergipe, Brasil. A identidade botânica da planta foi confirmada pelo biólogo Prof. Dr. Carlos Dias da Silva Jr e um exemplar coletado encontra-se depositado no Herbário da Universidade Federal de Sergipe (no. 008087) Universidade Federal de Sergipe, CCBS, Departamento de Biologia, São Cristovão, Sergipe, Brasil).

\section{Animais}

Foram utilizados camundongos Swiss (25-30 g) e cobaias (200-300 g) de ambos os sexos. Os animais foram mantidos em caixas plásticas com comida e água ad libitum, tendo sido submetidos à privação de ração durante as seis horas que precederam os experimentos. Os protocolos utilizados foram aprovados pelo Comitê de Ética em Pesquisa com Animais (CEPA) desta instituição (processos no. 39/05 e 50/06) e os experimentos foram realizados de acordo com as normas vigentes para trabalho com animais de laboratório (Andersen et al., 2004) e com as normas éticas para investigações de dor experimental em animais acordados (Zimmermann, 1983).

\section{Preparo do extrato}

As folhas da planta foram secas em estufa (40 ${ }^{\circ} \mathrm{C} ; 48 \mathrm{~h}$ ) e pulverizadas em moinho de facas. A infusão do pó das folhas foi preparada utilizando-se 70 gramas do pó por litro de água destilada $\left(100^{\circ} \mathrm{C}\right)$. Após filtração sob pressão reduzida, a solução obtida foi liofilizada (Liofilizador Benchtop, Vir Tis, USA) para a obtenção do extrato $\operatorname{seco}(7,4 \% ; 10,4 \mathrm{~g})$.

\section{Montagem da preparação in vitro (Madeira et al.,} 2002)

Os cobaios $(n=5)$, em jejum de aproximadamente $6 \mathrm{~h}$, foram sacrificados por concussão cerebral e exsanguinados. A porção terminal do intestino delgado foi retirada, dissecada e os segmentos $(1,5 \mathrm{~cm})$ foram montados em cubas de $10 \mathrm{~mL}$ para órgãos isolados em tampão Tyrode normal (composição em g/L: $\mathrm{NaCl} \mathrm{8,0;}$ $\mathrm{KCl} 0,2 ; \mathrm{CaCl}_{2} .2 \mathrm{H}_{2} \mathrm{O} 0,26 ; \mathrm{MgCl}_{2} .6 \mathrm{H}_{2} \mathrm{O} 0,09 ; \mathrm{NaHCO}_{3}$ 1,$0 ; \mathrm{NaH}_{2} \mathrm{PO}_{4} 0,05$ e glicose 1,$0 ; \mathrm{pH} 7,4 ; 37{ }^{\circ} \mathrm{C}$ ) sob aeração constante. A preparação isolada foi submetida a uma tensão inicial de $1 \mathrm{~g}$ e estabilizada durante $1 \mathrm{~h}$. As variações de tensão foram registradas através de um transdutor de força (Gould) acoplado a um sistema de aquisição de dados (Acquire 6600). Após a estabilização, foram construídas curvas concentração-resposta para concentraçãoes isoladas $(0,025 ; 0,050 ; 0,150 ; 0,5$; 1,5 e $2,0 \mathrm{mg} / \mathrm{mL}$ ) do extrato aquoso. As contrações induzidas por carbacol $(2 \mu \mathrm{M})$, histamina $(2 \mu \mathrm{M}) \mathrm{e} \mathrm{BaCl}_{2}$ $(0,03 \mathrm{M})$ foram também avaliadas na ausência e na presença do extrato aquoso de Phoradendron piperoides (1,5 mg/mL; pré-incubação, 20min).

\section{Estudo de toxicidade aguda $\left(\mathrm{DL}_{50}\right)$}

A toxicidade aguda em camundongos ( $\mathrm{n}=$ 5) foi avaliada através da administração oral de doses crescentes do extrato aquoso (1,3 e $5 \mathrm{~g} / \mathrm{kg}$ ), ou de igual volume do veículo (água). Após 48 horas, o número de óbitos foi verificado (Lorke, 1983).

\section{Estudos de analgesia}

Teste de contorções abdominais induzidas pelo ácido acético (Koster et al., 1959): Os animais $(\mathrm{n}=9)$ foram tratados com o extrato aquoso da planta nas doses de 100,200 e $400 \mathrm{mg} / \mathrm{kg}$ (v.o.) ou com igual volume de água (grupo controle; v.o.). Sessenta minutos após cada tratamento, foi administrado o ácido acético $(0,6 \% ; 0,1$ $\mathrm{mL}$ de solução/10 g de animal; i.p.) e as contorções abdominais foram contadas durante vinte minutos, iniciando-se dez minutos após a administração de ácido acético. O fármaco padrão morfina $(2,5 \mathrm{mg} / \mathrm{kg}$; i.p.; 45 minutos antes da administração do ácido acético) foi utilizado como controle positivo.

Teste da formalina (Hunskaar, Hole, 1987): Os animais foram tratados com o extrato aquoso da planta (100, 200 e $400 \mathrm{mg} / \mathrm{kg}$; v.o.), ou com igual volume de água destilada (v.o.). Sessenta minutos após cada tratamento, os camundongos $(\mathrm{n}=9)$ receberam uma injeção intraplantar de formalina a $1 \%(20 \mu \mathrm{L} /$ animal $)$. Após a administração da formalina, o tempo (s) que o animal passou lambendo a pata traseira esquerda foi cronometrado em duas fases (0-5 min primeira fase; 20-30 min; segunda fase), sendo considerado tempo zero o momento imediatamente após a administração da formalina. O fármaco padrão morfina $(7,5 \mathrm{mg} / \mathrm{kg}$, i.p., $45 \mathrm{~min}$ antes da formalina) foi utilizado como controle positivo.

Teste da placa quente (Ankier, 1974): Os animais $(\mathrm{n}=9)$ foram colocados sobre uma placa de 
Tabela 1. Efeito do extrato aquoso de Phoradendron piperoides no teste de contorções abdominais induzidas pelo ácido acético $(0,6 \%)$ em camundongos.

\begin{tabular}{|c|c|c|c|}
\hline Grupo & Dose $(\mathrm{mg} / \mathrm{kg})$ & No. de contorções (M \pm E.P.M.) & \% Redução ${ }^{a}$ \\
\hline Controle & - & $19,87 \pm 2,87$ & 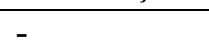 \\
\hline Extrato aquoso & 100 (v.o.) & $14,50 \pm 3,26$ & 27,1 \\
\hline Extrato aquoso & 200 (v.o.) & $17,75 \pm 1,87$ & 10,7 \\
\hline Extrato aquoso & 400 (v.o.) & $13,75 \pm 4,81$ & 30,8 \\
\hline Morfina & 2,5 (i.p.) & $1,23 \pm 0,25^{*}$ & 93,8 \\
\hline
\end{tabular}

* $\mathrm{p}<0,001$ (vs controle água; ANOVA, pós-teste de Bonferroni).a : porcentagem de redução do número total de contorções abdominais vs controle. $(n=9)$.

Tabela 2. Efeito do extrato aquoso (EA) de Phoradendron piperoides no teste da placa quente em camundongos.

\begin{tabular}{|c|c|c|c|c|}
\hline \multirow{2}{*}{ Tratamento / dose } & Tempo (min) & \multicolumn{2}{|c|}{$\mathrm{M} \pm \mathrm{EPM}$} & \multirow[b]{2}{*}{+60} \\
\hline & 0 & +15 & +30 & \\
\hline Controle & $8,98 \pm 0,84$ & $7,35 \pm 0,37$ & $9,50 \pm 1,00$ & $8,41 \pm 0,22$ \\
\hline EA (100 mg/kg; v.o.) & $7,31 \pm 0,75$ & $7,12 \pm 0,41$ & $8,52 \pm 0,40$ & $7,91 \pm 0,75$ \\
\hline EA (200 mg/kg; v.o.) & $8,07 \pm 1,11$ & $8,67 \pm 0,86$ & $8,35 \pm 1,03$ & $10,55 \pm 0,87$ \\
\hline EA (400 mg/kg; v.o.) & $9,74 \pm 0,43$ & $9,54 \pm 0,84$ & $9,77 \pm 0,57$ & $10,84 \pm 0,95$ \\
\hline Morfina (7,5 mg/kg; i.p.) & $30,00 \pm 0,00^{*}$ & $30,00 \pm 0,00 *$ & $30,00 \pm 0,00 *$ & $30,00 \pm 0,00 *$ \\
\hline
\end{tabular}

$* \mathrm{p}<0,001$ (vs controle; ANOVA, pós-teste de Bonferroni) $(\mathrm{n}=9$ )

Tabela 3. Efeito do extrato aquoso do Phoradendron piperoides no teste da formalina (1\%) em camundongos.

\begin{tabular}{llll}
\hline Grupo & $\begin{array}{l}\text { Dose } \\
(\mathrm{mg} / \mathrm{kg})\end{array}$ & $\begin{array}{l}1^{\text {a. }} \text { fase } \\
(\mathrm{M} \pm \mathrm{EPM})\end{array}$ & $\begin{array}{l}2^{\text {a. }} \text { fase } \\
(\mathrm{M} \pm \mathrm{EPM})\end{array}$ \\
\hline Controle & - & $53,33 \pm 4,55$ & $82,66 \pm 15,57$ \\
Extrato aquoso & 100 (v.o.) & $57,33 \pm 3,77$ & $55,66 \pm 12,84$ \\
Extrato aquoso & 200 (v.o.) & $47,22 \pm 4,92$ & $66,44 \pm 9,94$ \\
Extrato aquoso & 400 (v.o.) & $52,44 \pm 4,67$ & $78,44 \pm 11,83$ \\
Morfina & 7,5 (i.p.) & $6,57 \pm 2,02^{*}$ & $0,00 \pm 0,00^{*}$ \\
\hline
\end{tabular}

$* \mathrm{p}<0,001$ (vs controle; ANOVA, pós-teste de Bonferroni) $(\mathrm{n}=9)$

alumínio aquecida $\left(55 \pm 0,5{ }^{\circ} \mathrm{C}\right)$ e o tempo (s) que cada animal gastou para retirar uma pata traseira da placa de alumínio e levá-la à boca (tempo de latência) foi cronometrado. Foram realizadas medidas repetidas nos tempos $0,+15,+30$ e +60 minutos, sendo o tempo zero instituído como sendo 60 minutos após o tratamento com extrato aquoso $(100,200$ e $400 \mathrm{mg} / \mathrm{kg}$; v.o.) ou com igual volume de água destilada (v.o.). Com o objetivo de se evitar danos às patas dos animais foi estabelecido um tempo de corte de 30 segundos. Morfina $(7,5 \mathrm{mg} / \mathrm{kg}$; i.p.) foi administrada 45 minutos antes do tempo zero.

\section{Análise matemática dos dados}

Os resultados foram expressos através de média \pm erro padrão da média ( $\mathrm{M} \pm$ E.P.M.). $\mathrm{O}$ valor da $\mathrm{IC}_{50}$ foi estimado por interpolação (Alexander et al., 1999). Nos estudos de analgesia, as diferenças entre os grupos foram determinadas através da análise de variância ANOVA de uma via, seguida pelo teste de Bonferroni quando apropriado. Nos estudos in vitro, as diferenças entre os grupos foram determinadas através do teste $t$ pareado. Um nível de significância de $5 \%$ foi utilizado em todas as análises.

\section{RESULTADOS}

O extrato aquoso de Phoradendron piperoides não apresentou eficácia como analgésico em camundongos nas doses de 100, 200 e $400 \mathrm{mg} / \mathrm{kg}$ (p.o.) Nos testes das contorções abdominais induzidas por ácido acético, da placa quente e da formalina. Estes resultados encontram-se nas Tabelas 1-3.

No íleo isolado de cobaio, o extrato aquoso de $P$. piperoides $(0,05-2,0 \mathrm{mg} / \mathrm{mL}$; Figura 1$)$ promoveu 


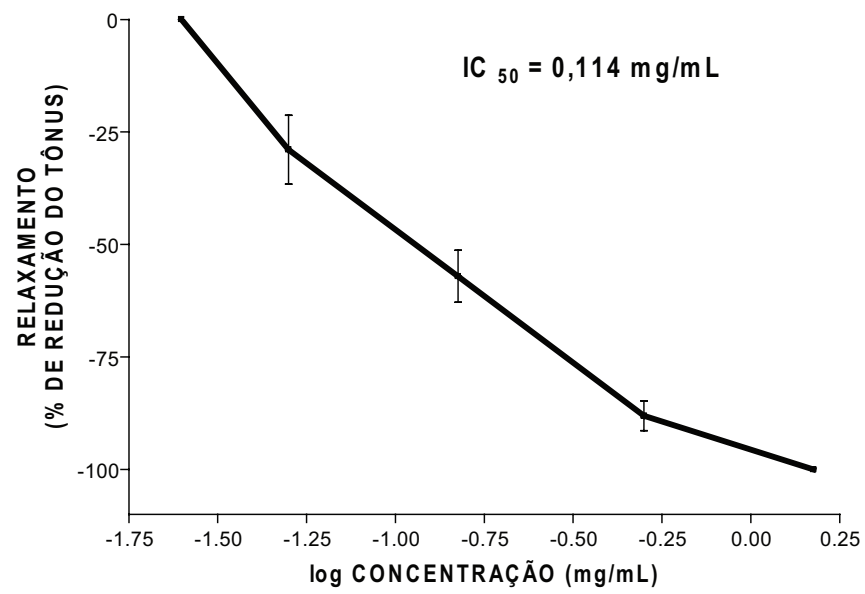

Figura 1. Efeito miorelaxante do extrato aquoso de Phoradendron piperoides em íleo isolado de cobaio.

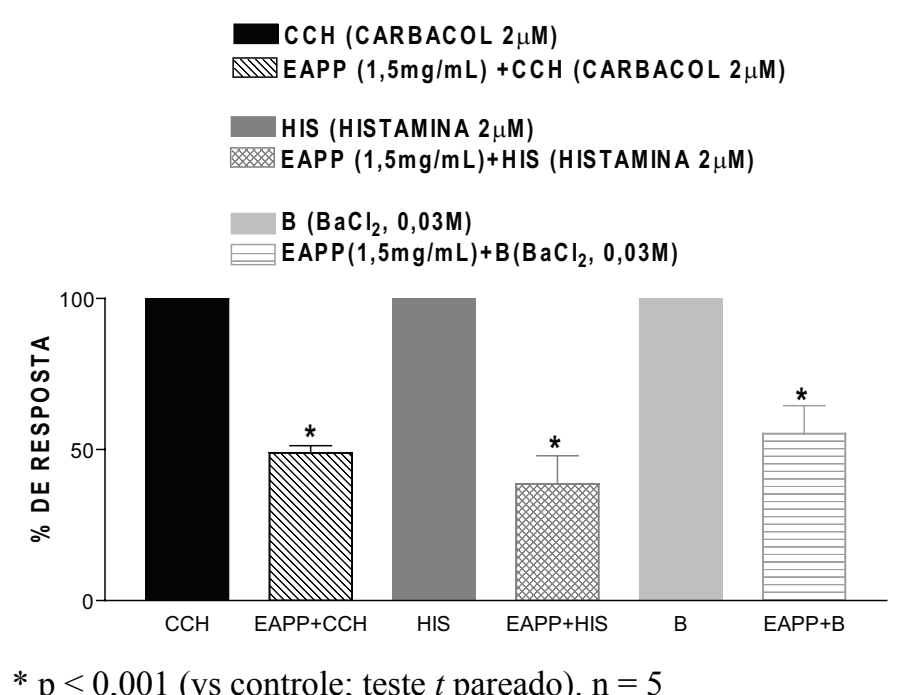

Figura 2. Efeito espasmolítico do extrato aquoso de Phoradendron piperoides (EAPP) em íleo isolado de cobaio.

relaxamento de forma concentração-dependente $\left(\mathrm{IC}_{50}=\right.$ $0,114 \mathrm{mg} / \mathrm{mL}$ ) e, na concentração de $1,5 \mathrm{mg} / \mathrm{mL}$, reduziu a amplitude da contração induzida por carbacol $(2 \mu \mathrm{M})$, histamina $(2 \mu \mathrm{M})$ e $\mathrm{BaCl}_{2}(0,03 \mathrm{M})$. As percentagens de redução observadas foram de 46,63 $\pm 2,21 \%(\mathrm{p}<0,001)$, $38,62 \pm 9,33 \%(\mathrm{p}<0,001)$ e $55,30 \pm 9,20 \%(\mathrm{p}<0,001)$ para o carbacol, histamina e $\mathrm{BaCl}_{2}$, respectivamente (Figura 2).

No ensaio de toxicidade aguda, não foi detectada a morte de nenhum animal com doses orais de até $5 \mathrm{~g} / \mathrm{kg}$. Também não foram observadas alterações de ordem comportamental ou autonômica.

\section{DISCUSSÃO}

No presente trabalho buscou-se inicialmente investigar o efeito analgésico sugerido pelo uso popular para o chá das folhas de Phoradendron piperoides. Este efeito foi avaliado em três modelos de estudo de nocicepção, denominados teste da placa quente, teste das contorções abdominais induzidas por ácido acético $(0,6 \%)$ e teste da formalina ( $1 \%)$. Porém, o extrato aquoso de $P$. piperoides, nas doses empregadas, não apresentou atividade analgésica em nenhum dos modelos de estudo de analgesia utilizados, vindo estes resultados a sugerir que este extrato não atue como analgésico (Koster et al., 
1959; Ankier, 1974; Hunskaar; Hole, 1987).

Por outro lado, fármacos antiespasmódicos são utilizados na terapêutica no combate à dor de origem espasmogênica. Considerando que o extrato aquoso de $P$. piperoides apresentou efeito miorelaxante e antiespasmódico torna-se razoável sugerir que o mesmo possa atuar no combate a este tipo de dor. O mecanismo preciso destes efeitos permanece por ser estabelecido. Porém, tanto as contrações induzidas por estímulos específicos (carbacol e histamina) quanto as contrações induzidas por estímulo inespecífico $\left(\mathrm{BaCl}_{2}\right)$ foram significativamente reduzidas pelo extrato aquoso, sendo este resultado compatível com uma atuação inespecífica.

Plantas da espécie Phoradendron são conhecidas por sua alta toxicidade (Hall et al., 1986; Spiller et al., 1996; Calzado-Flores et al., 2002). Contudo, no ensaio para toxicidade utilizado, o extrato aquoso da Phoradendron piperoides, até a dose de 5 $\mathrm{g} / \mathrm{kg}$, não provocou a morte de nenhum camundongo, sendo este resultado sugestivo que o extrato aquoso das folhas desta espécie apresente baixa toxicidade (Lorke, 1983).

Finalmente, cabe aqui ressaltar que, com o objetivo de se manter o estudo o mais próximo possível do uso popular da planta optou-se, no presente trabalho, pela utilização do extrato aquoso liofilizado preparado a partir da infusão das folhas da $P$. piperoides.

Em conclusão, os resultados obtidos indicam que o extrato aquoso de Phoradendron piperoides apresenta baixa toxicidade aguda e não apresenta efeito antinociceptivo em camundongos. Os efeitos miorelaxante e antiespasmódico observados podem, no entanto, ser responsáveis pelo uso da planta no combate à dor e parecem ser decorrentes de uma ação inespecífica.

\section{AGRADECIMENTOS:} financeiros.

Os autores agradecem ao CNPQ pelos auxílios

\section{REFERÊNCIAS}

Agra MF, França PF, Barbosa-Filho JM 2007. Synopsis of the plants known as medicinal and poisonous in Northeast of Brazil. Rev Bras Farmacogn 17: 114140 .

Alexander B, Browse DJ, Reading SJ, Benjamin IS 1999. A simple and accurate mathematical method for calculation of the EC50. J Pharm Toxicol Methods 41: 55-58.

Andersen ML, D'Almeida V, Ko MG, Kawakami R, Martins PJF, Magalhães LE, Tufik S 2004 Principios éticos e práticos do uso de animais de experimentação. São Paulo: UNIFESP.

Ankier SI 1974. New hot plate tests to quantify antinociceptive and narcotic-antagonist activities. Eur J Pharmacol

$$
\text { 27: 1-4. }
$$

Calzado-Flores C, Hurtado-Ramirez MB, Flores-Villanueva Z, Verde-Star MJ, Segura-Luna JJ, LozanoGarza G, Aguilar-Cuestas G 2002. Preliminary chronic toxicological study of aqueous extract of Phoradendron tomentosum. Proc West Pharmacol Soc 45: 162-163.

Gonzales E, Iglesias I, Carretero E, Villar A 2000. Gastric cytoprotection of bolivian medicinal plants. $J$ Ethnopharmacol 70: 329-333.

Hall AH, Spoerke DG, Rumack BH 1986. Assessing mistletoe toxicity. Ann Emerg Med 15: 1320-1323.

Hunskaar S, Hole K 1987. The formalin test in mice-dissociation between inflammatory and noninflammatory pain. Pain 30: 103-114.

Johansson S, Gullbo J, Lindholm P, Ek B, Thunberg E, Samuelsson G, Larsson R, Bohlin L, Claeson P 2003. Small, novel proteins from the mistletoe Phoradendron tomentosum exhibit highly selective cytotoxicity to human breast cancer cells. Cell $\mathrm{Mol}$ Life Sci 60: 165-175.

Kashiwada Y, Wang HK, Nagao T, Kitanaka S, Yasuda I, Fujioka T, Yamagishi T, Cosentino LM, Kozuka M, Okabe H, Ikeshiro Y, Hu CQ, Yeh E, Lee KH 1998. Anti-AIDS agents. 30. Anti-HIV activity of oleanolic acid, pomolic acid, and structurally related triterpenoids. J Nat Prod 61: 1090-1095.

Koster R, Anderson M, De Beer EJ 1959. Acetic acid for analgesic screening. Fed Proc 18: 412-416.

Li SS, Gullbo J, Lindholm P, Larsson R, Thunberg E, Samuelsson G, Bohlin L, Claeson P 2002. Ligatoxin $\mathrm{B}$, a new cytotoxic protein with a novel helix-turnhelix DNA-binding domain from the mistletoe Phoradendron liga. Biochem J 366: 405-413.

Lorke D 1983. A new approach to practical acute toxicity testing. Arch Toxicol 54: 275-287.

Madeira SVF, Matos FJA, Leal-Cardoso JH, Criddle DN 2002. Relaxant effects of the essential oil of Ocimum gratissimum on isolated ileum of the guinea pig. $J$ Ethnopharmacol 81: 1-4.

Queiroz-Neto A, Melito I 1990. Changes in sensitivity of the isolated guinea-pig vas deferens induced by a lyophilized Phoradendron latifolium leaf infusion. $J$ Ethnopharmacol 28: 183-189.

Spiller HA, Willias DB, Gorman SE, Sanftleban J. 1996. Retrospective study of mistletoe ingestion. $J$ Toxicol Clin Toxic 34: 405-408.

Timothy J. 1999. Boca Raton, Florida: CRC Press

Varela BG, Fernandez T, Ricco RA, Zolezzi PC, Hajos SE, Gurni AA, Alvarez E, Wagner ML 2004. Phoradendron liga (Gill. ex H. et A.) Eichl. (Viscaceae) used in folk medicine: anatomical, phytochemical, and immunochemical studies. J Ethnopharmacol, 94: 109-116.

Zimmermann M 1983. Ethical guidelines for investigations of experimental pain in conscious animals. Pain 16: 109-110. 